\title{
Quantized Layer Growth at Liquid-Crystal Surfaces
}

\section{Citation}

Ocko, B. M., A. Braslau, Peter S. Pershan, J. Als-Nielsen, and M. Deutsch. 1986. Quantized layer growth at liquid-crystal surfaces. Physical Review Letters 57(1): 94-97.

\section{Published Version}

doi:10.1103/PhysRevLett.57.94

\section{Permanent link}

http://nrs.harvard.edu/urn-3:HUL.InstRepos:10357487

\section{Terms of Use}

This article was downloaded from Harvard University's DASH repository, and is made available under the terms and conditions applicable to Other Posted Material, as set forth at http:// nrs.harvard.edu/urn-3:HUL.InstRepos:dash.current.terms-of-use\#LAA

\section{Share Your Story}

The Harvard community has made this article openly available.

Please share how this access benefits you. Submit a story.

\section{Accessibility}




\title{
Quantized Layer Growth at Liquid-Crystal Surfaces
}

\author{
B. M. Ocko, A. Braslau, and P. S. Pershan \\ Division of Applied Sciences, Harvard University, Cambridge, Massachusetts 02138 \\ J. Als-Nielsen \\ Ris@ National Laboratory, DK-4000 Roskilde, Denmark
}

and

M. Deutsch

Bar Ilan University, Ramat Gan, Israel

(Received 14 March 1986)

\begin{abstract}
We report $\mathrm{x}$-ray reflectivity measurements on the free surface of dodecylcyanobiphenyl (12CB) at the isotropic to smectic- $A$ phase transition. At about $10^{\circ} \mathrm{C}$ above $T_{I A}$, smectic- $A$-like ordering develops at the surface while the bulk phase remains isotropic. The angular dependence of the specular reflectivity is consistent with a sinusoidal density modulation, starting at the surface and terminating abruptly, after an integral number of bilayers. As the transition is approached the number of layers increases in quantized steps from zero to five before the bulk undergoes a firstorder transition to the smectic- $A$ phase.
\end{abstract}

PACS numbers: $64.70 . \mathrm{Md}, 68.10 .-\mathrm{m}$

The phase behavior of liquid-crystal systems is strongly influenced by the proximity of interfaces. Such effects can alter the orientational ordering and phase-transition temperatures, and, in some cases, new surface phases are introduced. In the isotropic phase of a nematogen, nematic ordering can be induced by a wall or free interface. For example, on cooling of the isotropic phase of pentylcyanobiphenyl (5CB) towards the first-order isotropic to nematic phase transition temperature $\left(T_{I N}\right)$, the penetration of the homeotropic nematic alignment induced by the surface diverges to produce an example of first-order orientational wetting. ${ }^{1-3}$ Below $T_{I N}$ the nematic phase completely wets the free surface. In the nematic phase, above the nematic to smectic- $A$ transition temperature, $T_{N A}$, the surface induces further positional (smectic) order that penetrates exponentially into the bulk nematic. ${ }^{4-5}$ Since the measured surface penetration length is exactly the same as the bulk correlation length and since the latter diverges as $T \rightarrow T_{N A}$, this is an example of critical wetting. In this paper we report measurements of the smectic ordering induced by the free surface in the isotropic phase of dodecylcyanobiphenyl (12CB), which does not have a nematic phase but undergoes a first-order transition from the isotropic to the smectic- $A$ phase at $T_{I A}=57.7^{\circ} \mathrm{C}$. As the temperature approaches $T_{I A}$ we observe five discrete transitions corresponding to the formation of single additional layers.

The layering transitions at the surface of a liquidcrystal phase closely resemble multilayer-physiadsorption phenomena on attractive substrates. ${ }^{6-10}$ For these phenomena the relative strengths of the ad- atom-adatom versus the adatom-substrate energies determine whether the condensed liquid forms droplets or completely wets the interface. For the wetting case, the layering may proceed discretely via the deposition of monolayers (krypton ${ }^{11}$ and ethylene on graphite ${ }^{12,13}$ ) or continuously if the interface to the vapor is rough (krypton on gold $^{14}$ ). The thickness of the wetting layer may diverge at coexistence (complete wetting) ${ }^{8,9,15}$ or remain finite (incomplete wetting). All of the adsorbed-gas problems suffer from the complications of a periodic substrate that interacts strongly with both the liquid and solid adsorbate phases. In the latter case, strains induced by differences in the two lattice parameters exclude the possibility of complete wetting. Since there is no substrate at a free surface of a liquid, the liquid-crystal problem does not suffer from this complication. One major difference between the liquid-crystal and the adsorbed-gas problems is that in the latter the physics can generally be studied as a function of both temperature and partial pressure, i.e., chemical potential, whereas for single-component liquid-crystal samples the temperature is the only free variable. Fortuitously, in mixtures, variations in the concentrations of homologs act as an additional field variable.

These experiments have been carried out by use of synchrotron radiation at HASYLAB at DESY in Hamburg, Germany. We present the essential technical details; however, a more comprehensive review can be found elsewhere. ${ }^{16}$ A germanium (111) monochromator crystal is set to Bragg reflect the bending magnet radiation at $\lambda=1.54 \AA$. The incident angle is adjusted by our tilting the monochromator down. This requires 
simultaneously adjusting the sample height. The spectrometer resolution, along the normal to the surface $\hat{\mathbf{z}}$, as determined by the divergence of the incoming beam, $\Delta q_{z}<10^{-3} \AA^{-1}$, is over an order of magnitude narrower than the width of the narrowest line shape. To account for the effects of bulk scattering and other nonspecular events, we have measured the intensity with the spectrometer misset from the specular condition. The background is structureless over the region of interest and is of the order of $2 \times 10^{-7}$ of the direct beam. The liquid-crystal sample was prepared by placement of $1 \mathrm{~g}$ of $12 \mathrm{CB}$, as received from $\mathrm{BDH}$, Ltd., on a silane-treated glass disk which is enclosed in a two-stage oven. This temperature was controlled to $\pm 0.002^{\circ} \mathrm{C}$ over several hours. The drift in $T_{I A}$ was less than $0.001{ }^{\circ} \mathrm{C} / \mathrm{h}$ if the temperature was kept within $1^{\circ}$ of $T_{I A}$.

The experiment is carried out by measurement of the specular reflectivity as a function of the wavevector transfer normal to the surface, $q_{z}{ }^{\prime}=(4 \pi / \lambda)$ $\times \sin (\alpha)$, at various temperatures in the isotropic phase of $12 \mathrm{CB}$, where $\alpha$ is the incident angle relative to the surface. We note that the $q$ vector in the material is $q_{z}=\left(q_{z}{ }^{\prime 2}-q_{c}{ }^{2}\right)^{1 / 2}$, where for 12CB $q_{c}=0.0215 \AA^{-1}$ is the critical $q$ vector for total external reflection, below which the reflectivity is close to $100 \%$. In Fig. 1, the reflectivity at $t=\left(T-T_{I A}\right) / T_{I A}$ $=6.3 \times 10^{-4}$ is plotted versus $q_{z}^{\prime} / q_{0}$, where $q_{0}=2 \pi / D=0.1605 \AA^{-1}$ is the smectic layering $q$ vector for $12 \mathrm{CB}$. The solid line, $R_{\mathrm{F}}\left(q_{z}^{\prime}\right)$, which corresponds to the theoretical reflectivity predicted from

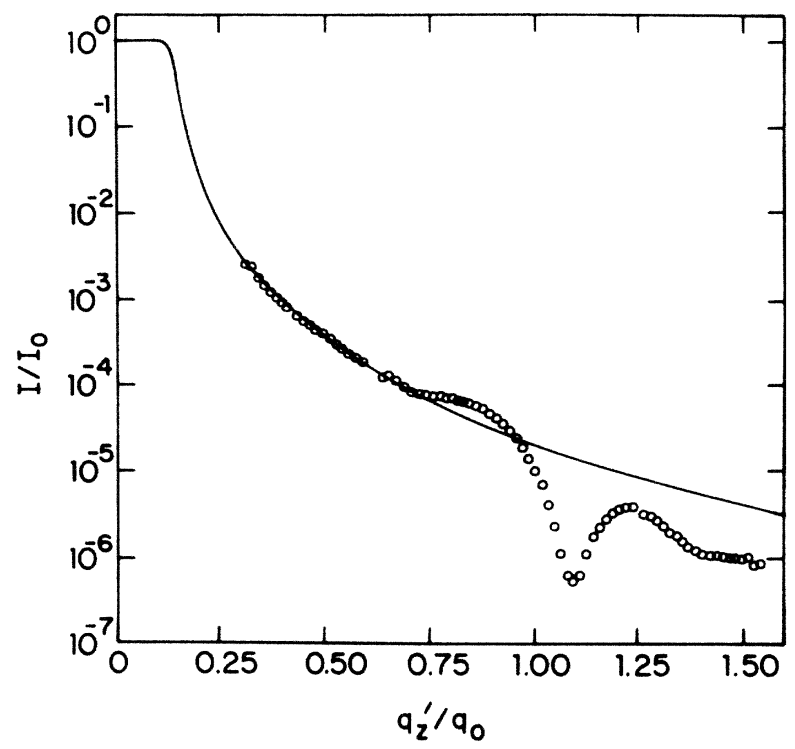

FIG. 1. The measured $x$-ray reflectivity from the surface of $12 \mathrm{CB}$ at $t=\left(T-T_{I A}\right) / T_{I A}=6.3 \times 10^{-4}$ is plotted vs $q_{z} / q_{0}$ as circles. The solid line is the theoretical Fresnel reflectivity for a step-function interface. the Fresnel law of optics for a simple step-function interface, agrees very well with the displayed data for all $q_{z}^{\prime} / q_{0}<0.50$. In Fig. 2 the reflectivity normalized to $R / R_{\mathrm{F}}$ is plotted versus $q_{z} / q_{0}$ at representative temperatures. At the highest temperature (Fig. 2, curve $f)$ the data are structureless, but as the transition is approached the profile becomes more complex corresponding to an increasing number of layers. At the lowest-temperature point (Fig. 2, curve $a$ ) the temperature is only $0.010^{\circ} \mathrm{C}$ above the smectic- $A$ phase and the profile may be smeared out by a small inhomogeneity in $T_{I A}$ or the temperature over the illuminated surface area. An essential feature of the data, over the temperature range $3 \times 10^{-4}<t<4$ $\times 10^{-3}$, is the nearly complete destructive interference at $q_{z} / q_{0}$ slightly greater than 1 .

The theoretical reflectivity for an arbitrary density

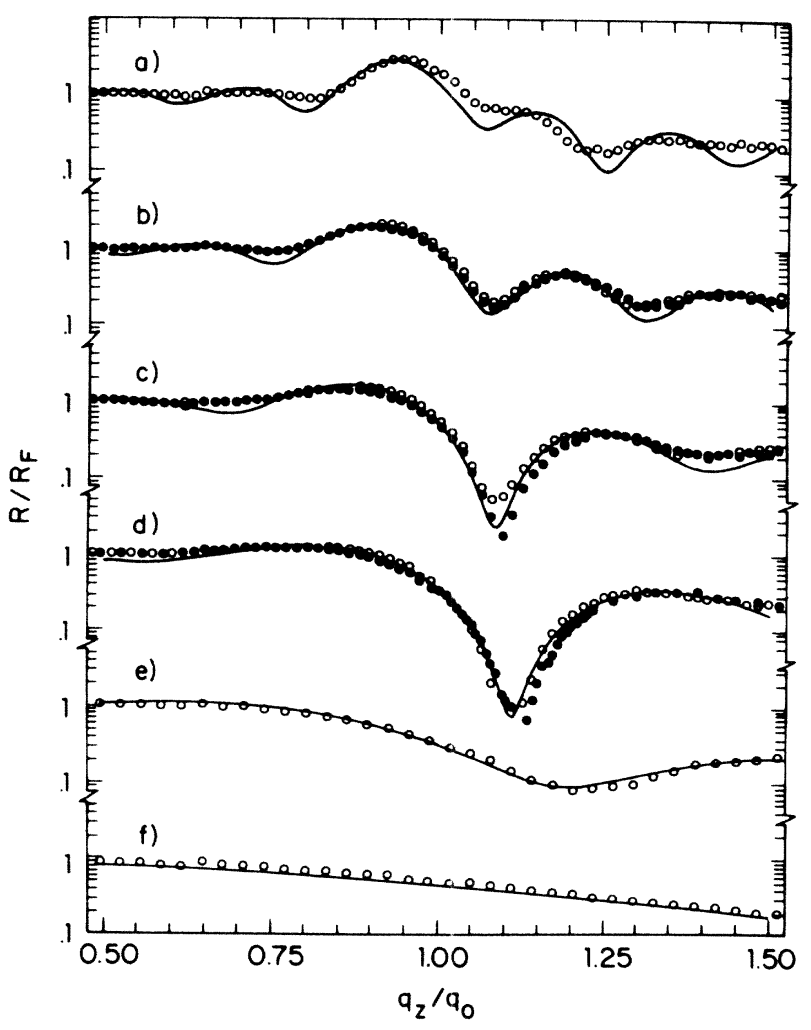

FIG. 2. The measured $x$-ray reflectivity in the isotropic phase, divided by the theoretical Fresnel reflectivity for a step interface, is plotted vs $q_{z} / q_{0}$ at curve $a, t=3 \times 10^{-5}$; curve $b, t=8 \times 10^{-5}$ (open circles) and $t=1.4 \times 10^{-4}$ (filled circles); curve $c, t=3.0 \times 10^{-4}$ (open circles) and $t=8.3$ $\times 10^{-4}$ (filled circles); curve $d, t=1.1 \times 10^{-3}$ (open circles) and $t=3.0 \times 10^{-3}$ (filled circles); curve $e, t=1.9 \times 10^{-2}$; and curve $f, t=6.1 \times 10^{-2}$. The solid lines are for a density model with a sinusoidal modulation terminated after an integral number of periods: five for curve $a$ through zero for curve $f$. 
distribution can be expressed in terms of $R_{\mathrm{F}}\left(q_{z}\right)$ :

$$
\frac{R\left(q_{z}\right)}{R_{\mathrm{F}}\left(q_{z}\right)}=\left|\frac{1}{\langle\rho\rangle} \int_{-\infty}^{\infty}\left\langle\frac{\partial \rho}{\partial z}\right\rangle e^{i q_{z} z} d z\right|^{2},
$$

in the limit of the Born approximation, ${ }^{4}$ i.e., $q_{z} \gg q_{c}$. The data can be modeled by a density function $\rho(z)$ that is obtained by a smoothed version of the function

$$
\rho(z)=\Theta\left(z-z_{0}\right)+H_{n}(z) B_{s} \sin (2 \pi z / D),
$$

where $\Theta\left(z-z_{0}\right)$ is a step function and $H_{n}(z)=1$ for $0<z<n D$ and zero elsewhere. The smoothing, due to thermal motion and finite atomic size, is represented by convolution of the two terms in $\rho(z)$ with Gaussian profiles. In Fig. 2, we show model curves (solid lines) for $n=0$ (bottom) through $n=5$ (top) with surface- and layer-smoothing Gaussian widths of 5.5 and $4.5 \AA$, respectively, a modulation amplitude $B_{s}=0.12$, and a phase factor $z_{0}=-0.35 D$. This phase corresponds to the peak value of the sine wave being displaced from the surface by $0.6 D$. The subsidiary maxima and minima above and below $q_{0}$ are a direct consequence of the sharp termination of the sine wave. For an exponential decay of the sine-wave amplitude, the reflectivity is Lorentzian in character with no subsidiary structure. ${ }^{4,5}$

At high temperatures $(n=0)$ the surface is well represented by a step function convoluted by a Gaussian with a width of $5.5 \AA$. This is the same form which describes the microscopic surface roughness of simple

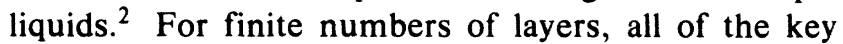
features of the data, including the intensities and widths of the primary peak and the positions of the peaks and dips, are well represented by the inclusion of a sine wave terminated after an integral number of layers. There are systematic deviations in the intensities of the subsidiary peaks and dips for $n=3,4$, and 5 which are not accounted for by this sharp-interface model. These deviations may result from a rough interface, although such features do not alter the determination of the number of layers.

In the bulk phase it has long been recognized that the key features of the smectic- $A$ phase are well represented by a single sinusoidal modulation. This is a valid approximation when there are a large number of layers, i.e., a small region in reciprocal space, and because of the effects of fluctuations in the molecular centers of mass. For the present case the surface fixes the centers of mass and the relevant region in reciprocal space is large, and it is therefore possible to estimate the electron density near the surface in terms of molecular models. ${ }^{17}$ This will be discussed more fully elsewhere $^{18}$; however, these results justify the present model in terms of a sinusoidal modulation.

Figure 3 displays the temperature dependence of the ratio $R / R_{\mathrm{F}}$ at the average peak position $q_{z}^{\prime}=0.15 \AA^{-1}$,

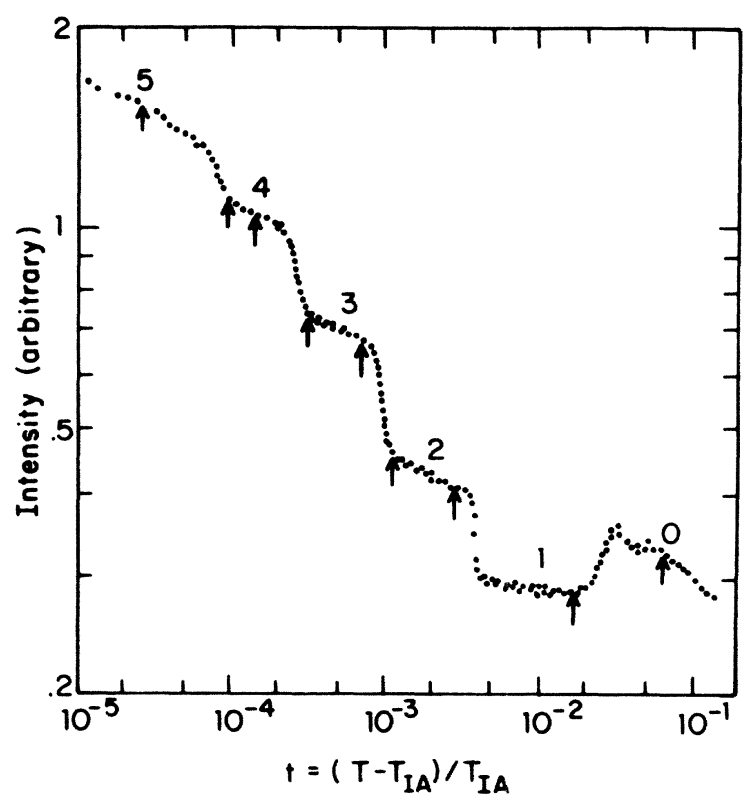

FIG. 3. The measured intensity at $q_{z}^{\prime}=0.15 \AA^{-1}$ in the isotropic phase upon heating. The quantized nature of the layering is clearly evident from the steplike nature of the intensity. The arrows correspond to the data sets plotted in Fig. 2.

obtained by slewing of the temperature. The slew rate ranged from $0.010^{\circ} \mathrm{C} / \mathrm{h}$ near the transition to $10.0^{\circ} \mathrm{C} / \mathrm{h}$ at the highest temperatures. The increase in the intensity at $t \geqslant 2.5 \times 10^{-2}$ is a consequence of the loss of destructive interference at $q_{z}^{\prime}=0.15 \AA^{-1}$ when the last layer melts.

The quantized nature of the layering is clearly evident from the steplike nature of the intensity shown in Fig. 3. From the data for the intensity versus $t$, one might expect that the first step upon cooling from the high-temperature surface phase would correspond to the formation of a single bilayer of smectic $A$. The analysis of the reflectivity profile supports this notion. Furthermore, each additional step corresponds to the growth of an additional bilayer. As the number of layers increases, the steplike nature of Fig. 3 becomes less distinct, and by five layers the layering transitions are no longer distinguishable. The layering transitions occur at temperatures $t_{0 \rightarrow 1}=(2.5 \pm 0.1) \times 10^{-2}$, $t_{1 \rightarrow 2}=(3.8 \pm 0.1) \times 10^{-3}, \quad t_{2 \rightarrow 3}=(9.6 \pm 0.3) \times 10^{-4}$, $t_{3 \rightarrow 4}=(2.5 \pm 0.2) \times 10^{-4}, \quad t_{4 \rightarrow 5}=(9.0 \pm 2.0) \times 10^{-5}$. The reduced range of each step, given by $\log \left(t_{n-1 \rightarrow n} / t_{n \rightarrow n+1}\right)$, increases as the number of layers decreases.

In the de Gennes model the coupling between the nematic and smectic order parameters greatly affects the behavior of the smectic correlation function for the nematic to smectic- $A$ transition. ${ }^{19}$ However, for the isotropic to smectic- $A$ transition there are no 
models for the smectic correlation function and there is no experimental evidence for smectic ordering in the bulk. In order to determine the effect of orientational ordering on the isotropic to smectic- $A$ transition we have also measured the surface ordering close to the nematic-smectic- $A$-isotropic triple point which occurs for nearly equal mixtures of $9 \mathrm{CB}$ and $10 \mathrm{CB}$. For $9.55 \mathrm{CB}$, which corresponds to $55 \%$ by weight $10 \mathrm{CB}$ and $45 \% 9 \mathrm{CB}$, the reflected intensity near $q_{0}$ decreases monotonically with temperature with no apparent signs of quantized layering. The reflectivity profiles do not exhibit subsidiary peaks centered at $q_{0}$. Therefore, we believe that the nearby nematic phase is responsible for a rougher interface between the smectic layers and the isotropic bulk. For triple-point wetting, the excess surface density, i.e., the smectic length, should diverge as $t \rightarrow 0 .{ }^{10}$ We do not believe that this can occur in the liquid-crystal problem since the smectic- $A$ correlation length is always finite along the nematic to smectic- $A$ line between the tricritical point $(9 \mathrm{CB})$ and the triple point $(9.45 \mathrm{CB}) .^{20}$ Along this line, as the triple point is approached, the transition becomes more first order ${ }^{21}$ and the correlation length decreases, ${ }^{20}$ therefore, complete wetting cannot occur at the isotropic to smectic- $A$ transition.

In conclusion, we note that although the free surface of the isotropic phase of $12 \mathrm{CB}$ is incompletely wetted by the smectic- $A$ phase, the partial wetting that does occur is discrete. The density profile normal to the surface is well described by a step function plus a surface sine wave terminated after an integer number of periods, convoluted by Gaussian smoothing functions. As the smectic- $A$ transition is approached, discrete layering transitions, up to five layers, are observed. There are no appropriate predictions which incorporate the effects of the nematic order parameter at the surface.

We would like to thank Carl Garland, Mehran Kardar, Michael Schick, and Jan Thoen for enlightening discussions; Robert Fiedenhansl, Francois Grey, and Mourits Nielsen for technical advice at DESY; and Dr. M. G. Pellatt (BDH, Ltd.) and Cyrus Safinya for providing the samples. This work was supported in part by grants from the Danish National Science Founda- tion, by the Risø National Laboratory, by the National Science Foundation through Grants No. DMR8212189, No. DMR 83-16979, and No. INT-83-11841, by the Joint Services Electronic Program through Grant No. N00014-84K-0465, and by the United States-Israel Binational Science Foundation.

${ }^{1}$ K. Miyano, Phys. Rev. Lett. 43, 51 (1979).

${ }^{2}$ D. Beaglehole, A. Braslau, M. Deutsch, P. S. Pershan, A. H. Weiss, J. Als-Nielsen, and J. Bohr, Phys. Rev. Lett. 54, 114 (1985).

${ }^{3}$ Ping Sheng, in Liquid Crystals and Ordered Fluids, edited by Julian F. Johnson (Plenum, New York, 1984), Vol. 4, p. 889.

4J. Als-Nielsen, F. Christensen, and P. S. Pershan, Phys. Rev. Lett. 48, 1107 (1982).

5P. S. Pershan and J. Als-Nielsen, Phys. Rev. Lett. 52, 759 (1984)

6J. G. Dash, Phys. Today 38, No. 12, 26-32 (1985).

${ }^{7}$ Rahual Pandit, M. Schick, and Michael Wortis, Phys. Rev. B 26, 5112 (1982).

${ }^{8}$ D. A. Huse, Phys. Rev. B 30, 1371 (1984).

${ }^{9}$ A. R. Lipowski, Phys. Rev. B 32, 1731 (1985).

${ }^{10}$ R. Pandit and M. E. Fisher, Phys. Rev. Lett. 51, 1772 (1983).

${ }^{11}$ A. Thomy and X. Duval, Appl. Opt. 21, 1894-1895 (1982).

${ }^{12}$ M. Sutton, S. G. J. Mochrie, and R. J. Birgeneau, Phys. Rev. Lett. 51, 407 (1983).

${ }^{13}$ M. Drir, H. S. Nham, and G. B. Hess, Phys. Rev. B 33, 5145 (1986).

14 J. Krim, J. G. Dash, and J. Suzanne, Phys. Rev. Lett. 52, 640 (1984).

15 John D. Weeks, Phys. Rev. B 26, 3998 (1982).

$16 \mathrm{~J}$. Als-Nielsen and P. S. Pershan, Nucl. Instrum. Methods 208, 545 (1983).

${ }^{17}$ E. F. Gramsbergen, W. H. de Jeu, and J. Als-Nielsen, J. Phys. (Paris), to be published.

18J. Als-Nielsen, A. Braslau, B. M. Ocko, and P. S. Pershan, to be published.

${ }^{19}$ P. G. de Gennes, Solid State Commun. 10, 753 (1972).

${ }^{20}$ B. M. Ocko, R. J. Birgeneau, J. D. Litster, and M. E. Neubert, Phys. Rev. Lett. 52, 208 (1984).

21 J. Thoen, H. Marynissen, and W. Van Dael, Phys. Rev. Lett. 52, 204 (1984). 